ORIGINAL ARTICLE

\title{
High postpartum rates of sexually transmitted infections among teens: pregnancy as a window of opportunity for prevention
}

\author{
J R Ickovics, L M Niccolai, J B Lewis, T S Kershaw, K A Ethier
}

Sex Transm Infect 2003;79:469-473

See end of article for authors' affiliations

.....................

Correspondence to: Jeannette R Ickovics, PhD,

Yale University,

Department of

Epidemiology and Public

Health, 135 College Street,

Suite 323, New Haven,

CT 06510, USA;

Jeannette.lckovics@

yale.edu

Accepted for publication 22 April 2003
Objectives: To identify incidence and predictors of Chlamydia trachomatis and Neisseria gonorrhoeae among postpartum adolescents. These estimates are compared to similar estimates among a cohort of non-pregnant, sexually active teens.

Methods: 203 pregnant and 208 non-pregnant adolescents aged 14-19 years were recruited from 10 community based health clinics in Connecticut, United States. Structured interviews and sexually transmitted infection (STI) testing using ligase chain reaction (LCR) were conducted at a baseline visit (during the third trimester for the pregnant adolescents), and at 6 and 12 month follow up visits ( 3 and 9 months post partum, for those pregnant at baseline).

Results: Among pregnant teens, new infections of $C$ trachomatis and $N$ gonorrhoeae increased from $7.1 \%$ at the 6 month follow up interview to $14.3 \%$ at the 12 month follow up interview; among non-pregnant teens, new infections remained relatively stable over the 6 and 12 month follow up interviews $19.0 \%$ to $8.3 \%$ ) (group by time interaction, $p=0.005$ ). C trachomatis and $N$ gonorrhoeae prevalence was 1.9 times higher (95\% Cl: 0.97 to $3.89, p=0.06$ ) among teens in the late postpartum follow up compared to the non-pregnant teens, controlling for baseline STIs. Predictors of postpartum STIs included having a new partner and number of partners per year of sexual activity.

Conclusions: Postpartum adolescents are vulnerable to STIs. Routine prenatal and postpartum care provide unique opportunities to promote condom use and other risk reduction interventions among adolescents. If sustained post partum, long term reproductive health can be promoted.
$\mathrm{T}$ he behaviours that put adolescents at risk for pregnancy also put them at risk for sexually transmitted infections (STIs). STI incidence and prevalence are higher among adolescent females than any other age and sex group, ${ }^{1-4}$ and they are particularly high among those who are pregnant. ${ }^{5}$ Although several studies have examined increased risk for STIs among adolescents during pregnancy, to date, no studies have focused specifically on postpartum STIs or examined STI rates prospectively from pregnancy into the postpartum period. Like pregnant adolescents, postpartum adolescents are at greater risk for STIs than the general population of adolescents because they often initiated sexual activity at an early age, remain sexually active, have more partners over the long course of childbearing years, and have increased biological susceptibility. ${ }^{6}$

STI prevention among pregnant adolescents has focused on screening for particular STIs before childbirth to prevent neonatal infection. However, it is also important to understand STI risk among postpartum adolescents who may become infected by a new partner or re-infected by a partner not treated during pregnancy. Moreover, decisions about postpartum contraception affect STI risk, even if not the primary motive. Prenatal care is often the first time adolescents address sexual health, and many are involved in routine care during and after pregnancy. Therefore, pregnancy and the postpartum period may provide critical windows of opportunity for clinic based interventions.

In a large retrospective cohort study of women who delivered infants in a mid-western hospital from 1992-8, Mahon et al documented rates of Chlamydia trachomatis and Neisseria gonorrhoeae infections 3 months post partum. ${ }^{8}$ Of 13086 eligible deliveries, $55 \%$ of women received postpartum STI testing; of these, $2.6 \%$ tested positive for $C$ trachomatis and
1.3\% tested positive for $N$ gonorrhoeae. This was the first population based study of postpartum STIs in the United States. However, the study was limited by its retrospective design, postpartum testing of only $55 \%$ of the women in the cohort, short term (that is, 3 month) follow up, and focus on a relatively low risk population.

The objective of the current study was to identify postpartum incidence and predictors of $C$ trachomatis and $N$ gonorrhoeae among adolescents in both the early and late postpartum period ( 3 and 9 months post partum). These estimates are compared to similar estimates among a cohort of non-pregnant, sexually active adolescents. C trachomatis and $N$ gonorrhoeae are the most common adolescent STIs and associated with serious sequelae; however, they can be diagnosed accurately and are curable using standard antibiotic regimens. ${ }^{8}$ This study extends previous literature by providing prospective, longitudinal data regarding STI infections measured using highly sensitive and specific nucleic acid amplification techniques during pregnancy and post partum among urban, high risk adolescents.

\section{METHODS}

Study sites and participants

Participants were recruited from 10 community based public health clinics in low income neighbourhoods of three Connecticut cities characterised by high incidence of adolescent pregnancy and STIs. ${ }^{9}{ }^{10}$ Patients served by these clinics are predominantly African American and Latina, with limited financial resources. Eligibility criteria for participation included being a female between the ages of 14 and 19 years, ever had sexual intercourse, nulliparous, HIV seronegative, and receiving obstetric or gynaecological care. 


\section{Procedures}

Participants were enrolled in a larger study examining STI risks from June 1998 to March 2000. Potentially eligible adolescents were approached by study staff, referred by healthcare providers, or responded to advertising materials. Recruitment was conducted sequentially to reach a target of at least 200 pregnant and 200 non-pregnant teens who met all inclusion criteria listed above.

Structured interviews were conducted in the third trimester of pregnancy or after clinic visits for non-pregnant teens 6 and 12 months later (window for follow up interviews was plus or minus 4 weeks). Instruments were pilot tested, modified for cultural and age appropriateness, and translated into Spanish. This study was approved by institutional review boards at Yale University and at participating clinics. Participants were paid \$25 for each interview.

\section{C trachomatis and $\mathbf{N}$ gonorrhoeae testing}

Urine samples were collected during each study visit and processed at a central laboratory. Testing for $C$ trachomatis and $N$ gonorrhoeae was conducted using ligase chain reaction (LCR; Abbott Laboratories, Chicago, IL, USA), which is highly sensitive and specific, with reported superiority to nonamplification techniques such as culture. ${ }^{11}{ }^{12}$ Following Burstein $e \mathrm{al}^{13}$ an incident STI was defined as a new infection more than 30 days after a documented positive or negative test result; thus insuring that each test result refers to a new infection. All patients with a positive $C$ trachomatis or $N$ gonorrhoeae test result were notified by telephone or mail and requested to return for treatment if not already provided in the clinic setting; they were also advised to refer their sexual partners for treatment.

\section{Measures}

Participants completed a structured, face to face interview that took 60-90 minutes to complete. The survey instrument was pilot tested for cultural and age appropriateness, and was translated into Spanish for Spanish speaking participants. Demographic, sexual history, and sexual risk behaviour variables were included as potential predictors of postpartum STIs.

\section{Demographic measures}

These included age (younger (14-16) $v$ older adolescents (1719 years)), race (Hispanic, black, white/other), and educational attainment (classified as in school or has a high school diploma $v$ not in school and no high school diploma).

\section{Sexual history variables}

These included age at first intercourse (age $\leqslant 14$ years $v$ $>14$ years), self reported history of a sexually transmitted disease (STD) ever (yes $v$ no), and number of sexual partners per year of sexual activity ( $\leqslant 1$ year $v>1$ year $)$.

\section{Sexual risk behaviours}

These included unprotected vaginal intercourse $(\geqslant 1 \quad v 0$ episodes of vaginal intercourse without a condom in the past 30 days), condom use during last sex act (no $v$ yes), the use of alcohol or other drugs before or during sex in the past 30 days (yes $v$ no), and current number of sexual partners (multiple $v$ single partner).

\section{Partnership specific factors}

They included presence of a new partner in the postpartum period (yes $v$ no), partner risk (yes $v$ no for history of an STD, sex with others in the past 6 months, or injection drug use ever), and partner age relative to participant ( $>2$ years older $v \leqslant 2$ years older).

\section{Statistical analysis}

The sample was described, stratified by pregnancy status. All proportions were compared using likelihood ratio $\chi^{2}$ tests. Logistic regression provided an estimate of the interaction between group (pregnant $v$ non-pregnant) and time. Odds ratios (OR) and 95\% confidence intervals (CI) were computed to compare the occurrence of new STI infections between the pregnant and non-pregnant groups at 6 and 12 month follow up interviews. Because all adolescents were screened and referred for STI treatment at baseline, infections detected at follow up were considered new infections and thus were directly comparable between the two groups.

Likelihood ratio $\chi^{2}$ tests were conducted to determine the association between baseline demographic, sexual history, sexual risk behaviour, and partnership specific factors with postpartum STIs at the 6 or 12 month visit. Based on these results, multivariate logistic regression was performed to identify the best set of predictors of postpartum STIs. Variables associated in the bivariate analyses with the presence of a postpartum STI where $\mathrm{p}<0.10$ were included for testing in the multivariate model. Associations between variables were estimated as ORs and were tested for significance with 95\% CIs. Statistical analyses were conducted using SPSS for Windows 10.0 (SPSS, Inc, Chicago, IL, USA).

\section{RESULTS}

Of 519 eligible adolescents, 203 pregnant and 208 nonpregnant adolescents were enrolled in the study $(81.2 \%$ of pregnant and $77.3 \%$ of non-pregnant adolescents who were eligible). The average age was 17.3 years, and the sample was predominantly black (44\%) and Hispanic (42\%). None of the participants was married. The study sample was representative of young women seen at the clinics with regard to race/ ethnicity, social class, and other demographic characteristics. Women in the study reported high baseline levels of sexual risk (for example, previous pregnancy, self reported history of previous STI, young age at first intercourse) (table 1).

Logistic regression analyses indicated a significant group by time interaction in combined rates of $C$ trachomatis and $N$ gonorrhoeae $(\mathrm{p}=0.005)$, whereby non-pregnant teens remained relatively stable over time; in contrast, pregnant teens had an increase in these STIs over time (fig 1). Most notably, among the teens pregnant at baseline, new infections of $C$ trachomatis and $N$ gonorrhoeae increased from $7.1 \%$ to $14.3 \%$ at the 6 and 12 month follow up interviews, respectively. Among non-pregnant teens, the proportion with new infections remained relatively stable over the 6 and 12 month follow up interviews (9.0 to 8.3\%). C trachomatis and $N$ gonorrhoeae prevalence was 1.94 times higher (95\% CI: 0.97 to $3.89, \mathrm{p}=0.061$ ) among teens 9 months post partum than among the comparison group of non-pregnant teens, controlling for baseline STI.

These analyses were restricted to those with LCR testing at all three time points ( 126 pregnant teens, 133 non-pregnant teens). Participants excluded from longitudinal analyses were more likely to be older (age 17-19 v 14-16 years) and more likely to have had a previous pregnancy (both $\mathrm{p}<0.05$ ). There were no differences between those included versus excluded in analyses with regard to any other demographic, sexual history, sexual risk behaviour, or relationship specific factor.

Post hoc analyses were conducted to determine if there was any bias associated with the timing of previous STI testing and diagnosis. We compared study participants pregnant versus non-pregnant at study entry with regard to differential screening and diagnosis in the 6 months before the baseline interview. For the pregnant teens, this would cover the entire period of their pregnancy. There was no difference in rates of STI screening based on medical record review: $87.2 \%$ 


\begin{tabular}{|c|c|c|c|}
\hline & $\begin{array}{l}\text { Pregnant } \\
(\mathrm{n}=203 \text { ) }\end{array}$ & $\begin{array}{l}\text { Non-pregnant } \\
(n=208)\end{array}$ & \\
\hline & No (\%) & No (\%) & $p$ Value \\
\hline \multicolumn{4}{|l|}{ Age (y) } \\
\hline $14-16$ & $83(40.9)$ & $98(47.1)$ & 0.20 \\
\hline $17-19$ & $120(59.1)$ & $110(52.9)$ & \\
\hline \multicolumn{4}{|l|}{ Race/ethnicity } \\
\hline Hispanic & $94(46.3)$ & 79 (38.2) & 0.08 \\
\hline African American & $88(43.3)$ & $92(44.4)$ & \\
\hline White/other & $21(10.3)$ & 36 (17.4) & \\
\hline \multicolumn{4}{|c|}{ In school/high school degree/GED } \\
\hline Yes & $156(76.8)$ & $185(88.9)$ & 0.002 \\
\hline No & 47 (23.2) & $23(11.1)$ & \\
\hline \multicolumn{4}{|l|}{ Previous pregnancy* } \\
\hline Yes & $108(53.2)$ & $50(24.0)$ & $<0.001$ \\
\hline No & $95(46.8)$ & $158(76.0)$ & \\
\hline \multicolumn{4}{|c|}{ History of an STI ever (self report) } \\
\hline Yes & $64(31.5)$ & $60(28.8)$ & 0.55 \\
\hline No & $139(68.5)$ & $148(71.2)$ & \\
\hline \multicolumn{4}{|c|}{ Age at first intercourse } \\
\hline$\leqslant 14$ & $85(41.9)$ & $110(53.1)$ & 0.02 \\
\hline$>14$ & $118(58.1)$ & $97(46.9)$ & \\
\hline \multicolumn{4}{|c|}{ Lifetime number of sexual partners } \\
\hline 1 & $57(28.1)$ & $47(22.6)$ & 0.44 \\
\hline $2-3$ & $72(35.5)$ & $79(38.0)$ & \\
\hline 4 & $74(36.5)$ & $82(39.4)$ & \\
\hline
\end{tabular}

$(n=177)$ of pregnant and $83.2 \%(n=173)$ of non-pregnant teens were tested in the 6 months before the baseline interview $(p=0.25)$. Furthermore, there were no differences in rates of STI diagnosis based on medical record review: $35.5 \%(\mathrm{n}=72)$ of pregnant and $36.1 \%(\mathrm{n}=75)$ of nonpregnant teens tested positive for either $C$ trachomatis or $N$ gonorrhoeae in the 6 months before the baseline interview $(\mathrm{p}=0.90)$. This latter finding was confirmed by state health department reports: $35.5 \%(\mathrm{n}=72)$ of pregnant and $38.9 \%$ $(\mathrm{n}=81)$ of non-pregnant teens were reported as positive for either $C$ trachomatis or $N$ gonorrhoeae in the 6 months before the baseline interview $(p=0.47)$. Finally, likelihood ratio $\chi^{2}$ tests among the pregnant adolescents with postpartum STI testing $(n=175)$ were conducted to identify potential predictors of postpartum STIs (table 2). Younger teens (aged 14-16 years) were marginally more likely to have a

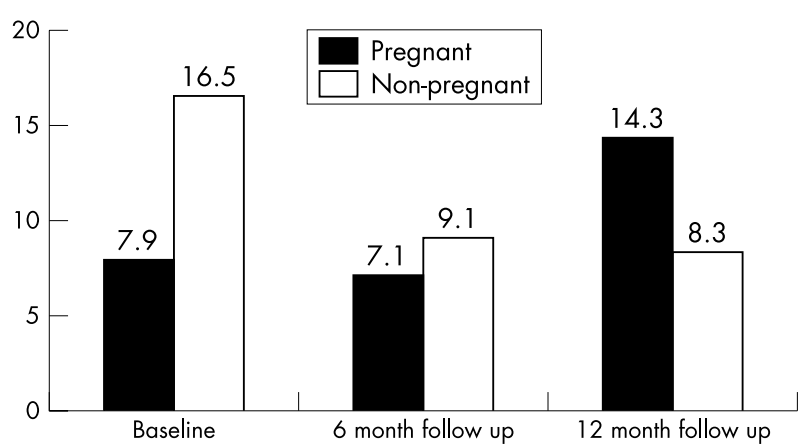

Figure 1 STI prevalence over time, stratified by baseline pregnancy status. Logistic regression for the group (pregnant $v$ non-pregnant) by time (baseline, 6 and 12 month follow up) interaction was statistically significant ( $p=0.005)$. C trachomatis and $N$ gonorrhoeae prevalence was 1.94 times higher $(95 \% \mathrm{Cl}$ : 0.97 to $3.89, p=0.06)$ among teens during the 12 month follow up (post partum) than among the comparison group of non-pregnant teens controlling for baseline STI. postpartum STI than older teens (aged 17-19 years). Among a wide array of behavioural characteristics tested, only four variables were at least marginally associated $(p<0.10)$ with a postpartum STI: number of current sex partners, average number of partners per year of sexual activity, having a new partner, and having a partner at risk. Final multivariate logistic regression was performed to identify the best set of predictors of postpartum STIs. Young women with new partners in the postpartum period were 6.3 times (95\% CI: 2.6 to 15.6 ) more likely to have a postpartum STI, and young women who had a greater number of partners per year of sexual activity were 3.0 times (95\% CI: 1.2 to 7.7 ) more likely to have a postpartum STI.

\section{CONCLUSIONS}

Nine months post partum, combined $C$ trachomatis and $N$ gonorrhoeae incidence was substantially higher than baseline and early (that is, 3 month) postpartum rates among pregnant adolescents. Furthermore, this was nearly double the incidence for non-pregnant adolescents. These results indicate that postpartum adolescents are particularly vulnerable to STI infection, most probably because of re-initiation of high risk sexual behaviour, including unprotected intercourse. Both the introduction of a new partner in the postpartum period and a history of multiple partners, representing behavioural trends towards risk, were influential in whether adolescents became infected. Considering one previous study of postpartum STIs among women of all ages, ${ }^{8}$ the rates documented in this study among teens are of serious concern: $3 \%$ of mid-western women tested positive for $C$ trachomatis and $N$ gonorrhoeae 3 months post partum compared to $7 \%$ of teens 3 months post partum and $14 \%$ of teens 9 months post partum. Postpartum adolescents are a high risk subgroup needing additional attention to ensure follow up screening and treatment.

Many adolescents, especially those with a history of pregnancy, are encouraged to use hormonal contraception (for example, long acting injectable depot medroxyprogesterone acetate (Depo Provera) $)^{14}$; although most effective at 


\begin{tabular}{|c|c|c|c|}
\hline & \multicolumn{2}{|l|}{ STD } & \multirow[b]{2}{*}{ p Value } \\
\hline & Yes $(n=31)$ & No $(n=144)$ & \\
\hline \multicolumn{4}{|l|}{ Demographics } \\
\hline \multicolumn{4}{|l|}{ Age (years) } \\
\hline $13-16$ & $18(58.1)$ & $57(39.6)$ & \multirow[t]{2}{*}{0.061} \\
\hline $17-19$ & $13(41.9)$ & $87(60.4)$ & \\
\hline \multicolumn{4}{|l|}{ Race } \\
\hline Black & $18(58.1)$ & $57(39.6)$ & \multirow{3}{*}{0.161} \\
\hline Hispanic & $8(25.8)$ & $58(40.3)$ & \\
\hline White/other & $5(16.1)$ & $29(20.1)$ & \\
\hline \multicolumn{4}{|l|}{ Education } \\
\hline Low & $7(22.6)$ & $32(22.2)$ & \multirow{2}{*}{0.965} \\
\hline High & $24(77.4)$ & $112(77.8)$ & \\
\hline \multicolumn{4}{|l|}{ Sexual history } \\
\hline Age (years) at first & & & \multirow{3}{*}{0.313} \\
\hline$\leqslant 14$ & $16(51.6)$ & $60(41.7)$ & \\
\hline$>14$ & $15(48.4)$ & $84(58.3)$ & \\
\hline \multicolumn{4}{|l|}{ History of an STD } \\
\hline Yes & $13(41.9)$ & $45(31.3)$ & \multirow[t]{2}{*}{0.258} \\
\hline No & $18(58.1)$ & $99(68.8)$ & \\
\hline \multicolumn{4}{|c|}{ Average number of sex partners per year of sexual activity } \\
\hline$>1$ & $24(77.4)$ & $75(52.1)$ & \multirow{2}{*}{0.008} \\
\hline$\leqslant 1$ & $7(22.6)$ & $69(47.9)$ & \\
\hline \multicolumn{4}{|c|}{ Sexual risk behaviour } \\
\hline Unprotected intercc & & & \multirow{3}{*}{0.895} \\
\hline Yes & $15(51.7)$ & $68(50.4)$ & \\
\hline No & $14(48.3)$ & $67(49.6)$ & \\
\hline \multicolumn{4}{|c|}{ Condom use last sex act } \\
\hline No & $22(71.0)$ & $117(81.3)$ & \multirow{2}{*}{0.214} \\
\hline Yes & $9(29.0)$ & $27(18.8)$ & \\
\hline \multicolumn{4}{|c|}{ Alcohol/drug use before sex $(n=144)$} \\
\hline Yes & $2(7.7)$ & $2(1.7)$ & \multirow{2}{*}{0.140} \\
\hline No & $24(92.3)$ & $116(98.3)$ & \\
\hline \multicolumn{4}{|c|}{ Number of current sex partners $(n=174)$} \\
\hline 0 & $4(12.9)$ & $11(7.7)$ & \multirow[t]{3}{*}{0.094} \\
\hline 1 & $20(64.5)$ & $118(82.5)$ & \\
\hline$\geqslant 2$ & $7(22.6)$ & $14(9.8)$ & \\
\hline \multicolumn{4}{|c|}{ Partnership specific factors } \\
\hline New partner & & & \\
\hline Yes & $14(45.2)$ & $16(11.1)$ & $<0.001$ \\
\hline No & $17(54.8)$ & $128(88.9)$ & \\
\hline Partner risk & & & \\
\hline Yes & $10(32.3)$ & $24(16.7)$ & 0.058 \\
\hline No & $21(67.7)$ & $120(83.3)$ & \\
\hline Age of partner relc & & & \\
\hline$>2$ years older & $16(55.2)$ & $59(49.2)$ & 0.561 \\
\hline$<2$ years older & $13(44.8)$ & $61(50.8)$ & \\
\hline
\end{tabular}

reducing risk for future pregnancy, it provides no protection against STIs, which results in a "contraceptive trade off dilemma." ${ }^{15}$ The more effective chosen contraceptives are for preventing pregnancy the less likely individuals are to use condoms to prevent STIs ${ }^{16}$; healthcare providers must seek to minimise the risk of both repeat pregnancy and STI.

This study is limited by use of a clinic based convenience sample, and some restrictions in statistical power as a result of the sample size and loss to follow up. Concerns regarding potential biases in differential rates of STI screening and diagnosis between the pregnant and non-pregnant teens were minimised by results of reanalyses that indicated no differences in these measures. Baseline differences, though not the focus of these analyses, were probably because the pregnant teens were in the third trimester of pregnancy and were significantly less likely to be sexually active, ${ }^{17}$ and therefore at reduced risk for STIs. Strengths of this study include an ethnically diverse sample recruited from 10 different hospitals and community health clinics serving young women at great risk for sexually transmitted infections. As part of the study protocol, all participants were screened multiple times for $C$ trachomatis and $N$ gonorrhoeae using nucleic acid amplification testing thus providing an objective biological outcome measure of STI incidence in the follow up period.

The results of this study indicate that postpartum adolescents should be targeted for STI prevention interventions, especially those with new partners and those with a greater number of partners. Routine prenatal and postpartum care provide unique opportunities to promote condom use and other risk reduction interventions among adolescents. However, the timing of these interventions may be critical because gaps can occur between obstetric care during pregnancy and gynaecological care in the later postpartum period. Moreover, after pregnancy, interventions may be more difficult because of the physical and emotional demands of infant care. None the less, many women, including adolescents, reduce health risk behaviours during pregnancy. ${ }^{18}$ If health enhancing behaviours are sustained post partum, long term reproductive health can be promoted.

\section{ACKNOWLEDGEMENTS}

This research was funded by the National Institute of Mental Health grant \#P01 MH/DA56826-01Al and training grant \#l T32 MH20031-02. 


\section{CONTRIBUTORS}

JI contributed to the study's design, served as principal investigator, participated in data analysis and interpretation, and wrote and revised the article; LN analysed and interpreted the data, wrote the statistical analyses and results sections of the article, and contributed to the article's revision; JL supervised data collection and management, and contributed to the article's revision; TK contributed to the data analysis and interpretation, and contributed to the article's revision; KE contributed to the study's design and to the article's revision.

\section{Authors' affiliations}

J R Ickovics, L M Niccolai, J B Lewis, T S Kershaw, Yale University, Department of Epidemiology and Public Health, Center for Interdisciplinary Research on AIDS, New Haven, CT, USA

K A Ethier, Centers for Disease Control and Prevention, Division of Sexually Transmitted Disease Prevention, Behavioral Interventions and Research Branch, Atlanta, GA, USA

\section{REFERENCES}

1 Berman SM, Hein K. Adolescents and STDs. In: Sexually transmitted diseases, 3rd ed. Holmes KK, et al. eds. New York: McGraw-Hill, 1999:129-42.

2 Burstein GR, Zenilman JM, Gaydos CA, et al. Predictors of repeat Chlamydia trachomatis infections diagnosed by DNA amplification testing among inner city females. Sex Transm Infect $2001 ; 77: 26-32$.

3 Whittington WLH, Kent $C$, Kissinger $P$, et al. Determinants of persistent and recurrent Chlamydia trachomatis infections in young women: results of a multicenter cohort study. Sex Transm Dis 2001;28:117-23.

4 Orr DP, Johnston K, Brizendine E, et al. Subsequent sexually transmitted infection in urban adolescents and young adults. Arch Pediatr Adolesc Med 2001 155:947-53.

5 Oh MK, Cloud GA, Baker SL, et al. Chlamydial infection and sexual behavior in young pregnant teenagers. Sex Transm Dis 1993;20:45-50.
6 Ickovics JR, Thayaparan B, Ethier K. Women and AIDS: contextual analysis. In: Handbook of health psychology. Baum A, Revenson T, Singer J, eds. Hillsdale, NJ: Earlbaum, 2000:821-39.

7 Whaley A. Preventing the high-risk sexual behavior of adolescents: focus on HIV/AIDS transmission, unintended pregnancy or both? J Adolesc Health 1999;24:378-82

8 Mahon BE, Rosenman MB, Graham MF, et al. Postpartum Chlamydia trachomatis and Neisseria gonorrhoeae infections. Am J Obstet Gynecol 2002; 186:1320-5.

9 Connecticut Department of Public Health, Vital Statistics Report. Hartford, CT, 1998.

10 Connecticut Department of Public Health, STD Control Program. Hartford, CT 2000.

11 Lee HH, Chernesky MA, Schachter J, et al. Diagnosis of Chlamydia trachomatis genitourinary infection in women by ligase chain reaction assay of urine. Lancet 1995:345:213-6.

12 Buimer M, Doornum GJ, Ching S, et al. Detection of Chlamydia trachomatis and Neisseria gonorrhoeae by LCR-based assays with clinical specimens from various sites: Implications for diagnostic testing and screening. J Clin Microbiol 1996:34:2395-400.

13 Burstein GR, Gaydos CA, Diener-West M, et al. Incident Chlamydia trachomatis infections among inner-city adolescent females. JAMA 1998;280:521-6.

14 Piccinino L, Mosher W. Trends in contraceptive use in the United States: 1982-1995. Family Planning Perspectives 1998;30:4-10.

15 Cates W. Contraception, unintended pregnancies, and sexually transmitted diseases: why isn't a simple solution possible? Am J Epidemiol 1996;143:311-18.

16 Darney P, Callegari LS, Swift A, et al. Condom practices of urban teens using Norplant contraceptive implants, oral contraceptives, and condoms for contraception. Am J Obstet Gynecol 1999;180:929-37.

17 Niccolai LM, Ethier KA, Kershaw TS, et al. Pregnant adolescents at risk: sexual behaviors and sexually transmitted disease prevalence. Am J Obstet Gynecol (in press).

18 Gilchrist LD, Hussey JM, Gillmore MR, et al. Drug use among adolescent mothers: prepregnancy to 18 months postpartum. J Adolesc Health $1996 ; 19: 337-44$.

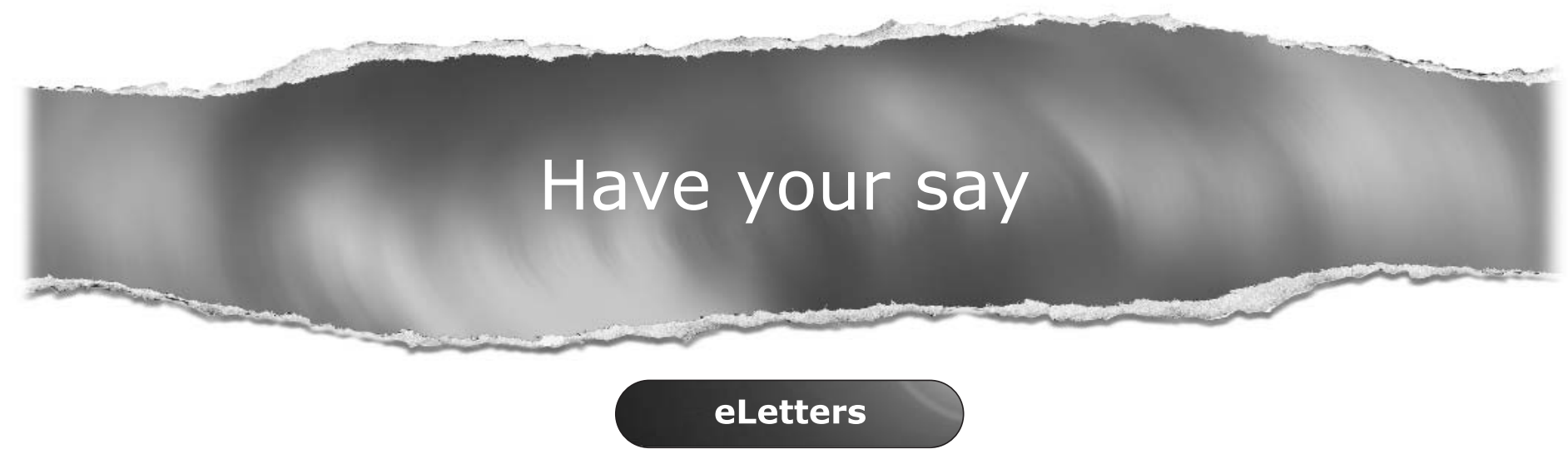

If you wish to comment on any article published in Sexually Transmitted Infections you can send an eLetter using the eLetters link at the beginning of each article. Your response will be posted on Sexually Transmitted Infections online within a few days of receipt (subject to editorial screening).

\section{www.sextransinf.com}

